Homology, Homotopy and Applications, vol.18(1), 2016, pp.339-342

\title{
A NOTE ON DESCENT FOR COALGEBRAS
}

\author{
MAURICE KIANPI AND CELESTIN NKUIMI-JUGNIA
}

(communicated by George Janelidze)

\begin{abstract}
Every natural transformation between endofunctors induces a functor between the categories of their coalgebras. We give sufficient conditions for that functor to preserve and reflect descent and effective descent morphisms.
\end{abstract}

\section{Introduction}

While a morphism in a finitely complete category is a descent morphism if and only if it is a pullback stable regular epimorphism, the characterization of effective descent morphisms is far more complicated, and in many concrete cases analysing its meaning can be challenging problem. Various relevant explanations are given by G. Janelidze and W. Tholen in their first expository article on descent theory [6], and more is added in their joint paper with M. Sobral [7]; let us also mention that the situation is somewhat simpler when the ground category is regular (see the same papers and related work of T. Everaert $[\mathbf{3}]$ ).

The present paper is related to the work of B. Mesablishvili [8], where he studies effective descent morphisms of (co)algebras over endofunctors. We study the functor between categories of such coalgebras induced by a natural transformation of the corresponding endofunctors, and show that it preserves and reflects descent and effective descent morphisms whenever the natural transformation is strongly monomorphic and cartesian.

\section{Coalgebras over endofunctors}

Let $\mathrm{C}$ be a category. For an endofunctor $F: \mathrm{C} \rightarrow \mathrm{C}$, a coalgebra is a pair $\mathcal{A}=$ $(A, \alpha: A \rightarrow F(A))$. A morphism from $(A, \alpha)$ to $(B, \beta)$ is a morphism $\varphi: A \rightarrow B$ such that $F(\varphi) \circ \alpha=\beta \circ \varphi$. With morphisms between them, coalgebras form a category $[\mathbf{1}, \mathbf{2}]$. Denote it by $\mathrm{C}_{F}$ and by $U: \mathrm{C}_{F} \rightarrow \mathrm{C}$ the forgetful functor. Although $U$ always reflect monos, it doesn't preserve them in general. The category $C_{F}$ has all colimits that exist in $C$ as well as all limits that are preserved by $F$. Thus, epis (isos) in $C_{F}$ are precisely morphisms which are epis (isos) in $C$ so that $U$ reflects epis (isos). In cases where either $\mathrm{C}$ is the category of sets or has epi-strong mono factorizations with $F$

Received August 29, 2012, revised January 21, 2015, September 1, 2015; published on May 18, 2016. 2010 Mathematics Subject Classification: 18A20, 18A23, 18A30, 18A32.

Key words and phrases: Category, (conservative) functor, pullback, coequalizer, preservation, reflection, (cartesian, natural) transformation, coalgebra, (effective) descent morphism.

Article available at http://dx.doi.org/10.4310/HHA.2016.v18.n1.a18

Copyright (C) 2016, International Press. Permission to copy for private use granted. 
preserving strong monos, strong monos in $C_{F}$ are morphisms which are strong monos in $C$ and $C_{F}$ has also epi-strong mono factorizations created by $U$, see $[\mathbf{1}]$.

Recall also, that a natural transformation of functors is called cartesian if so are all its naturality squares. Cartesian natural transformations are simply called cartesian transformations in $[4,5]$. Saying mono, or strong mono about a natural transformation, we mean a component-wise such.

Example 2.1. For all sets $A$ and $B, \eta: B \times(-)^{A} \rightarrow(B \times(-))^{A}$, where $\eta_{X}: B \times$ $X^{A} \rightarrow(B \times X)^{A}$ is defined by $\eta_{X}\langle b, \phi\rangle(a)=\langle b, \phi(a)\rangle$, is a cartesian transformation. It is mono unless $A=\emptyset \neq B$.

The following is easily proved:

Lemma 2.2. If $F \rightarrow G$ is mono and cartesian and $G$ preserves pullbacks, then so does $F$.

It is a routine exercise to prove the following theorem whose first statement can be found in $[\mathbf{9}]$ for $\mathrm{C}=$ Set.

Proposition 2.3. Let $F, G$ and $H$ be $C$-endofunctors. Every natural transformation $\eta: H \circ F \rightarrow G \circ H$ induces a functor $H_{\eta}: C_{F} \rightarrow C_{G}$ defined as $H_{\eta}(A, \alpha)=(H(A)$, $\left.\eta_{A} \circ H(\alpha)\right)$ and maps every morphism $\varphi:(A, \alpha) \rightarrow(B, \beta)$ into $H(\varphi): H(A) \rightarrow H(B)$. If $H$ is faithful, then so is $H_{\eta}$ and $H_{\eta}$ reflects monos; if in addition $\eta$ is mono, then for all $(A, \alpha)$ and $(B, \beta)$ in $C_{F}$ and every morphism $\varphi: A \rightarrow B$ in $C$ such that $H(\varphi):\left(H(A), \eta_{A} \circ H(\alpha)\right) \rightarrow\left(H(B), \eta_{B} \circ H(\beta)\right)$ is a morphism in $C_{G}, \varphi$ is a morphism in $C_{F}$ from $(A, \alpha)$ to $(B, \beta)$. If $H$ is conservative, i.e., $H$ reflects isos, then so is $H_{\eta}$. If $H$ preserves (reflects) epis, then so does $H_{\eta}$.

From now on, $H=I d, \widetilde{\eta}$ denotes $I d_{\eta}$ and $\widetilde{\eta}\left(C_{F}\right)$ the image of $C_{F}$ under $\widetilde{\eta}$.

Proposition 2.4. If $\eta$ is strong mono, then $\widetilde{\eta}\left(C_{F}\right)$ is closed under codomains of epis whose domains are contained in it. If $\eta$ is cartesian, then for every morphism $\varphi:\left(A, \alpha^{\prime}\right) \rightarrow \widetilde{\eta}(\mathcal{B})$ in $C_{G}$, there exists a unique morphism $\alpha: A \rightarrow F(A)$ such that $\alpha^{\prime}=\eta_{A} \circ \alpha$ and $\varphi:(A, \alpha) \rightarrow \mathcal{B}$ is a in morphism $C_{F}$. In particular, $\widetilde{\eta}\left(C_{F}\right)$ is closed under domains of morphisms whose codomains are contained in it.

Proof. Assume that $\eta$ is strong mono and let $\varphi: \widetilde{\eta}(\mathcal{A}) \rightarrow \mathcal{C}$ be an epi in $C_{G}$ where $\mathcal{C}=\left(C, \gamma^{\prime}\right)$ and $\mathcal{A}=(A, \alpha)$. Then $\eta_{C} \circ F(\varphi) \circ \alpha=\gamma^{\prime} \circ \varphi$. Now $\eta_{C}$ is a strong mono. Thus, there exists a unique $\gamma: C \rightarrow F(C)$ such that $\gamma \circ \varphi=F(\varphi) \circ \alpha$ and $\eta_{C} \circ \gamma=\gamma^{\prime}$, so that $\mathcal{C}=\widetilde{\eta}((C, \gamma))$. Assume that $\eta$ is cartesian and let $\varphi:\left(A, \alpha^{\prime}\right) \rightarrow \widetilde{\eta}((B, \beta))$ be a morphism in $\mathrm{C}_{G}$. Then $\eta_{B} \circ \beta \circ \varphi=G(\varphi) \circ \alpha^{\prime}$ and the universal property of the pullback yields a unique $\alpha: A \rightarrow F(A)$ such that $F(\varphi) \circ \alpha=\beta \circ \varphi$ and $\alpha^{\prime}=\eta_{A} \circ \alpha$.

\section{Preservation and reflection of (effective) descent morphisms}

Given an object $B \in \mathrm{C}$, the slice category $(\mathrm{C} \downarrow B)$ is the one whose objects are pairs $(A, \varphi)$, where $\varphi: A \rightarrow B$ is a morphism in C, and whose morphisms are $\psi:(A, \varphi) \rightarrow$ $\left(A^{\prime}, \varphi^{\prime}\right)$, where $\psi: A \rightarrow A^{\prime}$ is a morphism in $\mathrm{C}$ such that $\varphi^{\prime} \circ \psi=\varphi$. 
When $C$ has pullbacks, every morphism $\gamma: E \rightarrow B$ in $\mathrm{C}$ induces a functor $\gamma$ ! : (C $\downarrow$ $E) \rightarrow(C \downarrow B)$ sending $\varphi: A \rightarrow E$ to $\gamma \circ \varphi: A \rightarrow B$. This functor has a right adjoint $\gamma^{*}:(C \downarrow B) \rightarrow(C \downarrow E)$ (known as change-of-base functor) given by pulling back along $\gamma$. Denoting by $\operatorname{Des}(\gamma)$ the Eilenberg-Moore category of algebras of the monad induced by the adjunction $\gamma$ ! $\dashv \gamma^{*}$, we recall the following (e.g. from $[\mathbf{6}]$ ):

Definition 3.1. If the category $C$ has pullbacks, then a morphism $\gamma: E \rightarrow B$ is said to be:

(a) a descent morphism if the pullback functor $\gamma^{*}:(\mathrm{C} \downarrow B) \rightarrow(\mathrm{C} \downarrow E)$ is premonadic, i.e. if the comparison functor $\Phi^{\gamma}:(C \downarrow B) \rightarrow \operatorname{Des}(\gamma)$ is fully faithful;

(b) an effective descent morphism if $\gamma^{*}$ is monadic, i.e. if $\Phi^{\gamma}$ is an equivalence of categories.

The observations made in Section 2 give an easy proof of our main result:

Theorem 3.2. Assume that $C$ has pullbacks, $G$ preserves pullbacks and $\eta$ is mono and cartesian. Then $\widetilde{\eta}$ reflects and preserves (effective) descent morphisms.

Proof. From the assumptions imposed on $C$ and $G$, it follows that $F$ preserves pullbacks by Lemma 2.2, and, the categories $C_{F}$ and $C_{G}$ have pullbacks. By Proposition 2.3, the functor $\widetilde{\eta}$ is full and faithful. Moreover, by Proposition 2.4, $\widetilde{\eta}\left(\mathrm{C}_{F}\right)$ is closed under domains of morphisms whose codomains are contained in it. Therefore, for every morphism $\gamma:(E, \varepsilon) \rightarrow(B, \beta)$ in $C_{F}$, the comparison functor $\Phi^{\gamma}:\left(C_{F} \downarrow\right.$ $(B, \beta)) \rightarrow \operatorname{Des}(\gamma)$ can be identified (up to category equivalence) with the comparison functor $\Phi^{\widetilde{\eta}(\gamma)}:\left(\mathrm{C}_{G} \downarrow \widetilde{\eta}((B, \beta))\right) \rightarrow \operatorname{Des}(\widetilde{\eta}(\gamma))$. Hence $\widetilde{\eta}$ preserves and reflects descent and effective descent morphisms.

\section{Acknowledgments}

The authors thank George Janelidze for many helpful discussions, suggestions and comments that have helped to significantly improve this article.

\section{References}

[1] J. Adámek, Introduction to coalgebra, Theory Appl. Categ. 14 (2005), no. 8, 157-199.

[2] J. Adámek, H.-E. Porst, On varieties and covarieties in a category, Math. Structures Comput. Sci. 13 (2003), 201-232.

[3] T. Everaert, Effective descent morphisms of regular epimorphisms, J. Pure Appl. Algebra 216 (2012), 1896-1904.

[4] H.P. Gumm, On coalgebras and type transformations, Discuss Math. Gen. Algebra Appl. 27 (2007), 187-197.

[5] H.P. Gumm, On minimal coalgebras, Appl. Categ. Structures 16 (2008), no. 3, 313-332.

[6] G. Janelidze, W. Tholen, Facets of descent, I, Appl. Categ. Structures 2 (1994), 245-281. 
[7] G. Janelidze, M. Sobral, W. Tholen, Beyond Barr exactness: effective descent morphisms, in: M.C. Pedicchio, W. Tholen (Eds), Categorical Foundations: Special Topics in Order, Topology, Algebra and Sheaf Theory, in: Encyclopedia Math. Appl., vol. 97, Cambridge University Press (2004), 359-405.

[8] B. Mesablishvili, Descent in categories of (co)algebras, Homology Homotopy Appl. 7 (2005), no. 1, 1-8.

[9] J.J.M.M. Rutten, Universal coalgebra: a theory of systems, Theoret. Comput. Sci. 249 (2000), 3-80

Maurice Kianpi mkianpi@uy1.uninet.cm

Department of Mathematics, University of Yaounde I, Faculty of Science, PO Box 812, Yaounde, Cameroon

Celestin Nkuimi-Jugnia cnkuimi@uy1.uninet.cm

Department of Mathematics, University of Yaounde I, Faculty of Science, PO Box 812, Yaounde, Cameroon 